\title{
Analytic solutions of the linear Boltzmann transport equation for multilayered systems
}

\author{
Sui-Pin Chen and Ching-Ray Chang \\ Center of Nanostorage Research and Department of Physics, National Taiwan University, 10617 Taipei, Taiwan
}

(Received 2 February 2005; revised manuscript received 29 June 2005; published 24 August 2005)

\begin{abstract}
Instead of hard solving the linear response Boltzmann transport equation in the relaxation time approximation to study electron transport, an equivalent solution for the electron distribution can be easily determined by use of the effective mean-free-path model proposed in this paper. In the effective mean-free-path model, the electron distribution is related to the effective mean free path, and the difference between the effective mean free path and the mean free path is attributed to the effective diffusive scattering in the system. Given boundary conditions, the effective diffusive scattering can be specified. The process to determine the effective diffusive scattering in the effective mean-free-path model can be transformed into a diagrammatical method with a clear physical insight. This determination can be easily written down and is always explicitly expressed in analytic forms. Therefore, the analytic solutions for the electron distributions can be obtained in multilayered systems with various shapes and with realistic boundary conditions. All solutions derived from the effective mean-freepath model can be proven equivalent to those obtained by use of solving the complicated coupled equations from the linear response Boltzmann transport equation in the relaxation time approximation.
\end{abstract}

DOI: 10.1103/PhysRevB.72.064445

PACS number(s): 72.15.Lh, 73.50.Bk, 68.35.Fx, 72.25.Mk

\section{INTRODUCTION}

Spintronics has attracted much attention for its potential applications, particularly for the magnetic information storage technology. It began with two discoveries in the giant magnetoresistance (GMR) effects for a magnetic multilayer, which consists of a sequence of thin magnetic layers separated by equally thin nonmagnetic metallic layers. ${ }^{1-3}$ The two discoveries in the GMR imply (i) that the spin orientation of an electron is conserved over a distance up to several tens of nanometers, which is greater than the thickness of a typical magnetic multilayer, and (ii) that scattering is spin-dependent. ${ }^{3}$ When the spin orientation of an electron is conserved during the electron transport without spin-flip scattering, the main factor that determines the GMR is the spin asymmetry in scattering. That is to say, spin-dependent scattering is crucial to the GMR effect.

Usually, a common theory to specify spin-dependent scattering is based on the Boltzmann transport equation. A detail theory of spin-dependent interfacial scattering based on the Boltzmann transport equation was worked out by Camley and Barnas, ${ }^{4}$ and it was extended to include spin-dependent scattering in the bulk of the magnetic layer. ${ }^{5}$ Also, Levy et al. considered both spin-dependent interfacial and bulk scattering but used a more microscopic description based on the quantum-mechanical Kubo formula. ${ }^{6}$ Under some appropriate conditions, the results obtained from the Boltzmann and Kubo formalisms are essentially equivalent. ${ }^{3,7,8}$ However, the Green's function derivation of the Kubo formula is usually complicated and subtle. ${ }^{9}$ In contrast, the semiclassical Boltzmann formalism is flexible and versatile, which is quite suitable for treating the MR in most cases of practical interest. ${ }^{8}$ Indeed, the Boltzmann formalism provides a common approach to studying the electron transport in a finite system, such as the conductivity, ${ }^{10,11}$ the interfacial resistance, ${ }^{12-14}$ the giant magnetoresistance, ${ }^{4-17}$ the anisotropic magnetoresistance, ${ }^{18}$ and the spin accumulation. ${ }^{14,19}$

As a phenomenological formalism, the Boltzmann formalism treats all physical conditions for the investigated sys- tem as corresponding boundary conditions. That is, a more realistic theoretical system entails more complicated boundary conditions, and the corresponding set of coupled boundary equations become more sophisticated. But for the practical calculation, it is necessary to oversimplify the boundary conditions for a magnetic multilayer. ${ }^{4,12,13,15-18}$ These simplified boundary conditions may at least contain one of the following assumptions: (a) all mean free paths are the same and spin-independent; (b) the $F 1-M$ and the $F 2-M$ interfaces are nonreflective interfaces; (c) the incident angle is equal to the transmitted angle without considering the electron refraction law; (d) no in-scattering term at interfaces arises; (e) the outer surfaces of the $F 1$ and the $F 2$ layers are perfect reflective surfaces. Therefore, the difficulty in the Boltzmann formalism, or the Boltzmann transport equation model, is that on the one hand the solution for the electron distribution may be solved for simplified boundary conditions, but on the other hand more complicated boundary conditions are necessary for a realistic theoretical system. Besides, even though the boundary conditions are simplified, it is still difficult to determine either analytic or numerical solutions for a magnetic multilayer by use of the Boltzmann transport equation model. The only analytic solution presented by Liu and Xing is for the ferromagnet-metal-ferromagnet (F1-M-F2) trilayer with the same simple boundary conditions as given by Camley and Barnas, ${ }^{4,17}$ but their expressions are too complex to be applied to any realistic system. For other structures such as a cylindrical wire, ${ }^{18}$ a granular solid sphere, ${ }^{20}$ and an array of magnetic granules, ${ }^{21}$ only approximate results can be determined. To settle the above difficulties, the effective mean-free-path model equivalent to the Boltzmann transport equation model is developed in this paper.

The effective mean-free-path model involves the relations among the electron distribution, the effective mean free path, the mean free path, the effective diffusive scattering, and the boundary diffusion parameter, which are for a system with various boundary diffusive effects under an applied electric 
field. The electron distribution is linearly related to the effective mean free path, which was proposed by Rijks et al. ${ }^{18}$ Then, the difference between the effective mean free path and the mean free path is attributed to all possible induced effective diffusive scatterings in this paper. The effective diffusive scattering can be specified by the diffusion parameters and the mean free paths in an investigated system. For a single diffusive boundary, the effective diffusive scattering on the transport electron depends on the diffusion parameter at the diffusive boundary, and decays exponentially with the distance between the diffusive boundary and the transport electron. The decaying length of the effective diffusive scattering is characterized by the mean free path. In structures with more than one diffusive boundary, the determination to specify all total effective diffusive scatterings is associated with the sum of their individual effective diffusive scatterings from all diffusive boundaries. Moreover, the process to determine the total effective diffusive scattering in the effective mean-free-path model can be transformed into a diagrammatical method with a clear physical insight. The solutions from the diagrammatical way can be directly and easily written down, and are always explicitly expressed in analytic forms.

To clearly understand the expressions obtained from the effective mean-free-path model without loss of generality, in the beginning we take some simplified boundary conditions for the investigated systems. Finally, we will prove that the effective mean-free-path model can be applied to multilayered systems with various shapes and with realistic boundary conditions. In Sec. II, we give a brief review for the linear response Boltzmann transport equation in the relaxation-time approximation. Then this equation is applied to layered structures with boundary conditions. In Sec. III, we propose and introduce the effective mean-free-path model. In Sec. IV, we apply the effective mean-free-path model to the study of the trilayer structure with simple and complicated boundary conditions in Secs. IV A and IV B, respectively. Then, in Sec. IV C we conclude that the effective mean-free-path model is equivalent to the Boltzmann transport equation model, and extend the effective mean-free-path model to multilayered structures with realistic boundary conditions. We also investigate a solid sphere and an infinite thin cylindrical wire in Secs. V A and V B, respectively, and show that the effective mean-free-path model can be applied to structures with various shapes. Section VI is the conclusion.

\section{BOLTZMANN TRANSPORT EQUATION MODEL}

The Boltzmann transport equation model is generally described as follows. The general solutions for the electron distributions in structures with various shapes must be derived from the differential equation of the linear response Boltzmann transport equation in the relaxation time approximation. Imposing the given boundary conditions on the general solutions as well as their undetermined coefficients yields a set of coupled boundary equations. The undetermined coefficients can be determined only by use of solving this set of coupled boundary equations. When combined with spin-dependent scattering, the Boltzmann transport equation model becomes spin-dependent.

The linear response Boltzmann transport equation in the relaxation time approximation is given by ${ }^{4,18}$

$$
\mathbf{v}^{\sigma} \cdot \nabla_{\mathbf{r}} f_{\mathbf{v}}^{\sigma}(\varepsilon, \mathbf{r})-\mathbf{v}^{\sigma} \cdot e \mathbf{E} \frac{\partial f^{0, \sigma}(\varepsilon)}{\partial \varepsilon}=-\frac{f_{\mathbf{v}}^{\sigma}(\varepsilon, \mathbf{r})-f^{0, \sigma}(\varepsilon)}{\tau^{\sigma}} .
$$

The spin index $\sigma$ is $+1(-1,0)$ for spin-up (spin-down, spinindependent) state; the electron charge is $-e$; the applied electric field $\mathbf{E}$ is uniform, and the relaxation time $\tau^{\sigma}$ is spindependent. The electron distribution $f_{\mathbf{v}}^{\sigma}(\varepsilon, \mathbf{r})$, corresponding to the energy $\varepsilon$ and the velocity $\mathbf{v}$ at the position $\mathbf{r}$, can be divided into two parts: ${ }^{4}$ the Fermi-Dirac distribution $f^{0, \sigma}(\varepsilon)$ and the deviation distribution $g_{\mathbf{v}}^{\sigma}(\varepsilon, \mathbf{r})$, such that

$$
f_{\mathbf{v}}^{\sigma}(\varepsilon, \mathbf{r})=f^{0, \sigma}(\varepsilon)+g_{\mathbf{v}}^{\sigma}(\varepsilon, \mathbf{r}) .
$$

The spin-dependent Fermi-Dirac distribution $f^{0, \sigma}(\varepsilon)$ is isotopic and homogeneous, which is

$$
f^{0, \sigma}(\varepsilon)=\frac{1}{\exp \left[\beta\left(\varepsilon-\mu^{\sigma}\right)\right]+1},
$$

where the Boltzmann constant $k_{B}$ and the temperature $T$ are related by $\beta=1 / k_{B} T$. The energy of an electron is $\varepsilon$ $=1 / 2 m v^{2}$, where $m$ is the mass of an electron. The spindependent chemical potential $\mu^{\sigma}$ and the spin-independent chemical potential $\mu^{0}$ are related by $\mu^{\sigma}=\mu^{0}+\sigma h$, where $h$ is the molecular field of the magnetic material. The deviation distribution $g_{\mathbf{v}}^{\sigma}(\varepsilon, \mathbf{r})$ originates from all diffusive boundary effects under the driven electric field. It is anisotropic and nonhomogeneous, and can be expressed as

$$
g_{\mathbf{v}}^{\sigma}(\varepsilon, \mathbf{r})=\frac{\partial f^{0, \sigma}(\varepsilon)}{\partial \varepsilon}\left[\mathbf{v} \cdot e \mathbf{E} \tau^{\sigma}-\Delta \mu_{\mathbf{v}}^{\sigma}(\mathbf{r})\right]
$$

where $\Delta \mu_{\mathbf{v}}^{\sigma}(\mathbf{r})$ is the boundary chemical potential. Since the constraint of $\partial f^{0, \sigma} / \partial \varepsilon$ is a sharp peak around the Fermi energy, $\mathbf{v}$ turns to be the spin-dependent Fermi velocity $\mathbf{v}_{F}^{\sigma}$ restricted to the Fermi surface, and Eq. (4) becomes

$$
g_{\mathbf{v}}^{\sigma}(\varepsilon, \mathbf{r})=\frac{\partial f^{0, \sigma}(\varepsilon)}{\partial \varepsilon}\left[\mathbf{e}_{\mathbf{v}} \cdot e \mathbf{E} \lambda^{\sigma}-\Delta \mu_{\mathbf{v}}^{\sigma}(\mathbf{r})\right]
$$

where $\mathbf{e}_{\mathbf{v}}$ is a unit vector in the transport direction and $\lambda^{\sigma}$ $=v_{F}^{\sigma} \times \tau^{\sigma}$ is the mean free path. The notation $>(<)$ and $\mathbf{e}_{>(<)}$are used to represent to $\mathbf{v}$ and $\mathbf{e}_{\mathbf{v}}$ toward the forward (backward) boundary, respectively. Since the electron distribution $f_{\mathbf{v}}^{\sigma}(\varepsilon, \mathbf{r})$ in Eq. (2) with Eqs. (3) and (5) must satisfy Eq. (1), the boundary chemical potential $\Delta \mu_{\mathbf{v}}^{\sigma}(\mathbf{r})$ is valid under

$$
\mathbf{e}_{\mathbf{v}} \cdot \nabla_{\mathbf{r}}\left[\Delta \mu_{\mathbf{v}}^{\sigma}(\mathbf{r})\right]=-\frac{\left[\Delta \mu_{\mathbf{v}}^{\sigma}(\mathbf{r})\right]}{\lambda^{\sigma}} .
$$

In a layered structure, the direction perpendicular to the layer plane is set parallel to the $z$ direction, and then the general solution for $\Delta \mu_{\mathrm{v}}^{\sigma}(z)$ to Eq. (6) can be obtained; accordingly, the general solution for $f_{\mathbf{v}}^{\sigma}(\varepsilon, z)$ in Eq. (2) is 


$$
\begin{aligned}
f_{\mathbf{v}}^{\sigma}(\varepsilon, z)= & f^{0, \sigma}(\varepsilon)+\frac{\partial f^{0, \sigma}(\varepsilon)}{\partial \varepsilon} \mathbf{e}_{\mathbf{v}} \cdot e \mathbf{E} \lambda^{\sigma} \\
& \times\left[1-G_{\mathbf{v}}^{\sigma} \exp \left[\frac{-z}{\lambda^{\sigma} \cos \theta_{\mathbf{v}}}\right]\right],
\end{aligned}
$$

where $\mathbf{e}_{\mathbf{v}} \cdot \mathbf{e}_{z}=\cos \theta_{\mathbf{v}}$. The incident angle $\theta_{\mathbf{v}}$ can be divided into $\theta_{>}$and $\theta_{<}$such that $\theta_{>}$for $0 \leqslant \theta_{\mathbf{v}} \leqslant \pi / 2$ refers to the right transport direction, and $\theta_{<}=\left(\pi-\theta_{\mathbf{v}}\right)$ for $\pi / 2 \leqslant \theta_{\mathbf{v}} \leqslant \pi$ refers to the left transport direction. The positions of the right and the left boundaries in a layer are denoted $z_{>}$and $z_{<}$, respectively. So, $f_{\mathbf{v}}^{\sigma}(\varepsilon, z)$ becomes $f_{>(<)}^{\sigma}(\varepsilon, z)$ with $G_{>(<)}^{\sigma}$, $\mp\left(z-z_{<(>)}\right)$, and $\cos \theta_{>(<)} ; f_{>(<)}^{\sigma}(\varepsilon, z)$ is the electron distributions at the Fermi level corresponding to positive $(>)$ and negative $(<)$ group velocities in the current direction. Similarly, $g_{\mathbf{v}}^{\sigma}(\varepsilon, z)$ in Eq. (5) becomes $g_{>(<)}^{\sigma}(\varepsilon, z)$. The coefficient $G_{>(<)}^{\sigma}$ within $f_{>(<)}^{\sigma}(\varepsilon, z)$ and $g_{>(<)}^{\sigma}(\varepsilon, z)$ depends on the given boundary conditions for the transport electron.

The boundary conditions can be specified based on the conservation of the number of electrons, and contain the following variables: the diffusion parameter $D^{\sigma}$, the reflection parameter $R^{\sigma}$, the transmission parameter $T^{\sigma}$, the incident angle $\theta_{\mathbf{v}}$, the mean free path $\lambda^{\sigma}$, and the layer thickness. Take an $i-j$ bilayer as an example. The $i-j$ bilayer consists of the left $i$ layer and the right $j$ layer. Denote all physical variables by the subscripts $i-j, i-i$, and $j-j$ to indicate the interface between the $i$ layer and the $j$ layer, the outer surface of the $i$ layer, and the outer surface of the $j$ layer, respectively. At the $i-j$ interface, the relation between $R_{i-j}\left(\mathbf{v}_{i}, \mathbf{v}_{j}\right)$ and $T_{i-j}\left(\mathbf{v}_{i}, \mathbf{v}_{j}\right)$ is given by $R_{i-j}\left(\mathbf{v}_{i}, \mathbf{v}_{j}\right)+T_{i-j}\left(\mathbf{v}_{i}, \mathbf{v}_{j}\right)=S_{i-j}\left(\mathbf{v}_{i}, \mathbf{v}_{j}\right)=1$ $-D_{i-j}\left(\mathbf{v}_{i}, \mathbf{v}_{j}\right)$, where $R_{i-j}\left(\mathbf{v}_{i}, \mathbf{v}_{j}\right)=S_{i-j}\left(\mathbf{v}_{i}, \mathbf{v}_{j}\right) \times \alpha_{i-j}\left(\mathbf{v}_{i}, \mathbf{v}_{j}\right)$ and $T_{i-j}\left(\mathbf{v}_{i}, \mathbf{v}_{j}\right)=S_{i-j}\left(\mathbf{v}_{i}, \mathbf{v}_{j}\right) \times \beta_{i-j}\left(\mathbf{v}_{i}, \mathbf{v}_{j}\right)$. The $S_{i-j}\left(\mathbf{v}_{i}, \mathbf{v}_{j}\right)$ describes the degree of diffusiveness of the scattering. ${ }^{16,22}$ The nondiffusive reflection coefficient $\alpha_{i-j}\left(\mathbf{v}_{i}, \mathbf{v}_{j}\right)$ and the nondiffusive transmission coefficient $\beta_{i-j}\left(\mathbf{v}_{i}, \mathbf{v}_{j}\right)$ are known from the solution of the quantum mechanical scattering problem, and satisfy the relation $\alpha_{i-j}\left(\mathbf{v}_{i}, \mathbf{v}_{j}\right)+\beta_{i-j}\left(\mathbf{v}_{i}, \mathbf{v}_{j}\right)=1 .^{12,16,22}$ At the $i$ $-j$ interface, the boundary conditions for the transport electron associated with $g_{\mathbf{v}}^{\sigma}(\varepsilon, \mathbf{r})$ are given by ${ }^{4,12,16,22}$

$$
\begin{aligned}
& g_{j,>}=R_{i-j} g_{j,<}+T_{i-j} g_{i,>}, \\
& g_{i,<}=R_{i-j} g_{i,>}+T_{i-j} g_{j,<,},
\end{aligned}
$$

when $D_{i-j}=D_{j-i}, R_{i-j}=R_{j-i}$, and $T_{i-j}=T_{j-i}$ are assumed. Similarly, the boundary conditions at the right outer surface of the $j$ layer and the left outer surface of the $i$ layer are

$$
\begin{gathered}
g_{j,<}=R_{j-j} g_{j,>}, \\
g_{i,>}=R_{i-i} g_{i,<},
\end{gathered}
$$

respectively.

All terms in Eqs. (8)-(11) are sensitive to $\mathbf{v}$, and also the incident angle $\theta_{\mathbf{v}}=\theta_{i n}$, the reflected angle $\theta_{r}$, and the transmitted angle $\theta_{t}$. The relations among these angles have the effect of these boundary equations. Without in-scattering terms, these angles $\theta_{i n}, \theta_{r}$, and $\theta_{t}$ obey the electron reflection law and the electron refraction law for the oblique incidence. $^{22}$ But, with in-scattering terms, the relation among $\theta_{i n}, \theta_{r}$, and $\theta_{t}$ must be reconsidered. In this paper, we first set $\theta_{i n}=\theta_{r}=\theta_{t}$ in order to simplify the expressions. Then, we will discuss the influence of $\theta_{\text {in }} \neq \theta_{r} \neq \theta_{t}$.

\section{EFFECTIVE MEAN-FREE-PATH MODEL}

Rijks et al. proposed the concept of the effective mean free path $\lambda_{e f f, \mathbf{v}}^{\sigma}(\mathbf{r})$ that $\lambda_{e f f, \mathbf{v}}^{\sigma}(\mathbf{r})$ is proportional to the deviation distribution $g_{\mathbf{v}}^{\sigma}(\varepsilon, \mathbf{r}),{ }^{18}$ which means

$$
g_{\mathbf{v}}^{\sigma}(\varepsilon, \mathbf{r})=\frac{\partial f^{0, \sigma}(\varepsilon)}{\partial \varepsilon} \mathbf{e}_{\mathbf{v}} \cdot e \mathbf{E} \lambda_{e f f, \mathbf{v}}^{\sigma}(\mathbf{r}) .
$$

So the electron distribution $f_{\mathbf{v}}^{\sigma}(\varepsilon, \mathbf{r})$ in Eq. (2) becomes

$$
f_{\mathbf{v}}^{\sigma}(\varepsilon, \mathbf{r})=f^{0, \sigma}(\varepsilon)+\frac{\partial f^{0, \sigma}(\varepsilon)}{\partial \varepsilon} \mathbf{e}_{\mathbf{v}} \cdot e \mathbf{E} \lambda_{e f f, \mathbf{v}}^{\sigma}(\mathbf{r}) .
$$

Due to the same $g_{\mathbf{v}}^{\sigma}(\varepsilon, \mathbf{r})$, Eqs. (5) and (12) must be equivalent. Then, the diffusive boundary effects on $g_{\mathbf{v}}^{\sigma}(\varepsilon, \mathbf{r})$ can be specified by the boundary chemical potential of $\Delta \mu_{\mathbf{v}}^{\sigma}(\mathbf{r})$ in Eq. (6) in terms of Eq. (5) and also by $\lambda_{e f f, \mathbf{v}}^{\sigma}(\mathbf{r})$ in terms of Eq. (12). When $\Delta \mu_{\mathbf{v}}^{\sigma}(\mathbf{r})=0$ due to no diffusive boundary effects, $g_{\mathbf{v}}^{\sigma}(\varepsilon, \mathbf{r})$ in Eq. (5) reduces to $g_{\mathbf{v}}^{\sigma}(\varepsilon)$,

$$
g_{\mathbf{v}}^{\sigma}(\varepsilon)=\frac{\partial f^{0, \sigma}(\varepsilon)}{\partial \varepsilon} \mathbf{e}_{\mathbf{v}} \cdot e \mathbf{E} \lambda^{\sigma}
$$

This indicates that $\lambda_{e f f, \mathbf{v}}^{\sigma}(\mathbf{r})$ must return to $\lambda^{\sigma}$ in terms of $g_{\mathbf{v}}^{\sigma}(\varepsilon, \mathbf{r})$ in Eq. (12), which implies that the difference between $\lambda_{e f f, \mathbf{v}}^{\sigma}(\mathbf{r})$ and $\lambda^{\sigma}$ results from the influence of the diffusive boundary effects; the effective mean free path $\lambda_{e f f, \mathbf{v}}^{\sigma}(\mathbf{r})$ at a complex system is not equal to the mean free path $\lambda^{\sigma}$ at a homogeneous bulk is the existence of diffusive scatterings. Now, to construct the relation between the difference and the influence is a different way to specify the electron distribution $f_{\mathbf{v}}^{\sigma}(\varepsilon, \mathbf{r})$ in the investigated system. In this paper, we propose a model to construct the relations among all above physical variables. Since this model is based on the concept of the effective mean free path, it is named the effective mean-free-path model.

The solution in Eq. (14) is the electron distribution to the Drude model, in which the diffusive scattering within a bulk material on the transport electron can be completely and averagely specified by the mean free path $\lambda$. This solution is only appropriate to a single film without any boundary diffusive scattering and a bulk material. In contrast, the solution in Eq. (13) includes the diffusive boundary effects, and is appropriate for a system with more general collisions, such as those from the diffusive boundaries and those from the mismatch between two adjacent materials. Due to these general collisions, the corresponding effective mean free path $\lambda_{e f f}$ may be less than $\lambda$. Here, the relation between $\lambda_{e f f}$ and $\lambda$ is treated as

$$
\lambda_{e f f}=\lambda \times\left(1-D_{e f f}\right),
$$

where $D_{\text {eff }}$ specifies the effective diffusive scatterings from all diffusive boundaries.

Consider a system with only the single boundary in which the interfacial diffusion parameter is $D^{\sigma}$ at $\mathbf{r}_{b}$ in order to 


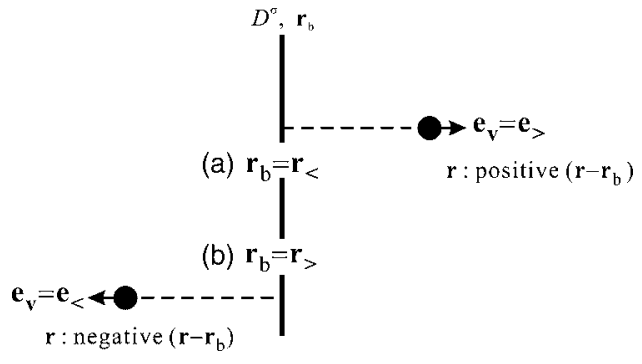

FIG. 1. The diffusive boundary with the diffusion parameter $D^{\sigma}$ is at the position $\mathbf{r}_{b}$, and the transport electron is at $\mathbf{r}$, where (a) $\mathbf{r}_{<}, \mathbf{e}_{>}$and (b) $\mathbf{r}_{>}, \mathbf{e}_{<}$are diagrammatically shown, respectively. The solid circle represents the transport electron, and the solid arrow indicates the direction of the transport electron.

determine the solution for $D_{\text {eff }}$ in Eq. (15). There are two physical constraints on the effective diffusive scattering. The first is that $D_{e f f}$ is $D^{\sigma}$ in the case of $\left|\mathbf{r}-\mathbf{r}_{b}\right|=0$, where $\mathbf{r}=\mathbf{r}_{b}$. The other is that $D_{\text {eff }}$ is limited to zero in the case of $\mid \mathbf{r}$ $-\mathbf{r}_{b} \mid \rightarrow \infty$, where the transport electron is very far from the diffusive boundary. According to these two extreme cases, it is reasonable to propose that $D_{\text {eff }}$ is a maximum of $D^{\sigma}$ at $\mathbf{r}$ $=\mathbf{r}_{b}$ and decays exponentially as the ratio of the distance $\mid \mathbf{r}$ $-\mathbf{r}_{b} \mid$ to the characteristic length of $\lambda^{\sigma}$ increases. This effective diffusive scattering $D_{e f f}$ is from $D^{\sigma}$ at $\mathbf{r}_{b}$ and is on the transport electron with $\mathbf{e}_{\mathbf{v}}$ at $\mathbf{r}$, and can be specified by

$$
D_{e f f}=D^{\sigma} \times \exp \left[\frac{-\left|\mathbf{r}-\mathbf{r}_{b}\right|}{\lambda^{\sigma}}\right]
$$

It is useful to define the traveling factor as

$$
t_{\mathbf{v}}^{\sigma}=\exp \left[\frac{-\left|\mathbf{r}-\mathbf{r}_{b}\right|}{\lambda^{\sigma}}\right]
$$

Equation (16) is also consistent with the calculation based on the real-space Kubo formula, which represents that the influence from the diffusive source on the transport electron decays exponentially as the distance $\left|\mathbf{r}-\mathbf{r}_{b}\right|$ increases. ${ }^{23}$ Moreover, there exists an important relation between $D_{\text {eff }}$ and the transport direction $\mathbf{e}_{\mathbf{v}}$. For the transport electron with $\mathbf{e}_{<}$and with $\mathbf{e}_{>}$, the same position of $\mathbf{r}_{b}$ is divided into $\mathbf{r}_{>}$and $\mathbf{r}_{<}$, respectively (Fig. 1). According to the current continuity, the transport electron with $\mathbf{e}_{>}$at $\mathbf{r}$ is related to the transport electron with $\mathbf{e}_{>}$at $\mathbf{r}-d \mathbf{r}$, and then to the transport electron with $\mathbf{e}_{>}$at $\mathbf{r}_{<}$. So, the effective diffusive scattering from the single boundary at $\mathbf{r}_{<}$is on the transport electron with $\mathbf{e}_{>}$not with $\mathbf{e}_{<}$[Fig. 1(a)], and vice versa [Fig. 1(b)].

Usually, complex are real systems, such as a multilayered structure with more than one diffusive boundary. For example, there are two nonreflective diffusive boundaries for a transport electron with $\mathbf{e}_{>}$, which are specified by $D^{\sigma}$ at $\mathbf{r}_{<}$ and $D^{\prime \sigma}$ at $\mathbf{r}_{<}^{\prime}$ in Fig. 2(a). According to Eqs. (16) and (17) as well as the current continuity, this total effective diffusive scatterings on the transport electron with $\mathbf{e}_{>}$at $\mathbf{r}$ can be specified by the sum of

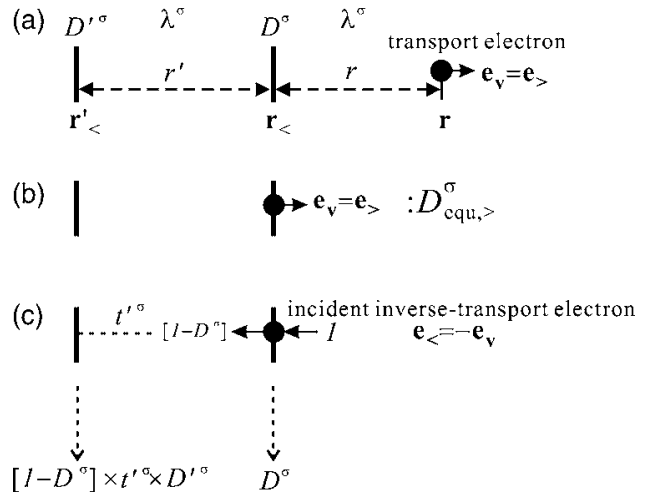

FIG. 2. Layered structure with the two diffusive boundaries which are specified by the diffusion parameters $D^{\sigma}$ at $\mathbf{r}_{<}$and $D^{\prime \sigma}$ at $\mathbf{r}_{<}^{\prime}$, respectively (a). The equilibrium diffusion parameter $D_{e q u,>}^{\sigma}$ is on the transport electron with the transport direction $\mathbf{e}_{>}$and at the position $\mathbf{r}_{<}$(b). For the determination of $D_{e q u,>}^{\sigma}$ in a diagrammatical method (c), an incident inverse-transport electron with the transport direction $\mathbf{e}_{<}$is injected from $\mathbf{r}_{<}$, and the traveling factor is $t^{\prime \sigma}$. The solid circle represents the transport electron, and the solid arrow indicates the direction of the transport electron. The spin-dependent mean free path is $\lambda^{\sigma}$.

$$
D^{\sigma} \times \exp \left[\frac{-r}{\lambda^{\sigma}}\right],
$$

from the first diffusive boundary with $D^{\sigma}$ at $\mathbf{r}_{<}$and

$$
\left[1-D^{\sigma}\right] \times D^{\prime \sigma} \times \exp \left[\frac{-\left(r+r^{\prime}\right)}{\lambda^{\sigma}}\right],
$$

from the second diffusive boundary with $D^{\prime \sigma}$ at $\mathbf{r}_{<}^{\prime}$ through the first diffusive boundary. The $r\left(r+r^{\prime}\right)$ is the distance between the boundary at $\mathbf{r}_{<}\left(\mathbf{r}_{<}^{\prime}\right)$ and the transport electron with $\mathbf{e}_{>}$at $\mathbf{r}$. The sum of Eqs. (18) and (19) specifies these total effective diffusive scatterings, which are

$$
D_{e f f}=D_{e q u,>}^{\sigma} \times \exp \left[\frac{-r}{\lambda^{\sigma}}\right]
$$

and

$$
D_{e q u,>}^{\sigma}=D^{\sigma}+\left[1-D^{\sigma}\right] \times D^{\prime \sigma} \times \exp \left[\frac{-r^{\prime}}{\lambda^{\sigma}}\right] .
$$

The equilibrium diffusion parameter $D_{e q u,>}^{\sigma}$ is $D_{e f f}$ with $r$ $=0 ; D_{e q u,>}^{\sigma}$ specifies the all effective diffusive scatterings on the transport electron with $\mathbf{e}_{>}$at $\mathbf{r}=\mathbf{r}_{<}$[Fig. 2(b)].

By inspection, the process to determine $D_{e q u,>}^{\sigma}$ in Eq. (21) can be transformed into a diagrammatical method by use of injecting an incident inverse-transport electron into the system [Fig. 2(c)]. The incident inverse-transport electron is injected from $\mathbf{r}_{<}$and is along $-\mathbf{e}_{>}=\mathbf{e}_{<}$[Fig. 2(c)]. The value of $D_{e q u,>}^{\sigma}$ in Eq. (21) is on a unit transport electron with $\mathbf{e}_{>}$at $\mathbf{r}_{<}$, and is equal to the determination to specify the total diffusive scattering on this incident inverse-transport electron. The total diffusive scattering on this injected electron is the superposition of individual effective diffusive scatterings from all transport paths, which are analyzed as follows. This injected unit electron with $\mathbf{e}_{<}$from $\mathbf{r}_{<}$is first scattered at the 
first diffusive boundary with $D^{\sigma}$ at $\mathbf{r}_{<}$. So, the first effective diffusive scattering on it is specified by $D^{(1)}=1 \times D^{\sigma}$. Next, the electron flux of the transmitted transport electron is [1 $\left.-D^{\sigma}\right]$ rather than 1 , and travels a distance $r^{\prime}$ with a traveling factor $t^{\prime \sigma}$, but is scattered at the second diffusive boundary with $D^{\prime \sigma}$ at $\mathbf{r}_{<}^{\prime}$. So, the second effective diffusive scattering from this transport path, which is on the unit incident transport electron with $\mathbf{e}_{<}$injected from $\mathbf{r}_{<}$, is specified by $D^{(2)}$ $=\left[1-D^{\sigma}\right] \times t^{\prime \sigma} \times D^{\prime \sigma}$. Then, the residual transmitted transport electron undergoes no diffusive scattering due to no more diffusive boundary. Finally, the determination to specify the total effective diffusive scatterings from all transport paths is $D^{(1)}+D^{(2)}$, and is easily confirmed to be equal to $D_{\text {equ },>}^{\sigma}$ in Eq. (21).

Based on the above descriptions about the diagrammatical method, the equilibrium diffusion parameter $D_{\text {equ,v }}^{\sigma}$, which is on a unit transport electron with the transport direction $\mathbf{e}_{\mathbf{v}}$ at $\mathbf{r}_{b}$, can be determined by use of injecting an incident inversetransport electron into the system. This unit incident inversetransport electron is along the transport direction $-\mathbf{e}_{\mathbf{v}}=\mathbf{e}_{-\mathbf{v}}$ and is injected from $\mathbf{r}=\mathbf{r}_{b}=\mathbf{r}_{-\mathbf{v}}$. Associated with the superposition of all effective diffusive scatterings on this electron, $D_{e q u, v}^{\sigma}$ can be obtained. Since the total effective diffusive scatterings from more than one diffusive boundary on the transport electron with $\mathbf{e}_{\mathbf{v}}=\mathbf{e}_{>(<)}$at $\mathbf{r}=\mathbf{r}_{<(>)}$is specified by the equilibrium diffusion parameter $D_{e q u, \mathbf{v}}^{\sigma}=D_{e q u,>(<)}^{\sigma}$ instead of the interfacial diffusion parameter $D^{\sigma}$, the effective mean free path in Eq. (15) can be extended as

$$
\lambda_{e f f,>(<)}^{\sigma}(\mathbf{r})=\lambda^{\sigma}\left[1-D_{e q u,>(<)}^{\sigma} \times \exp \left[\frac{-\left|\mathbf{r}-\mathbf{r}_{<(>)}\right|}{\lambda^{\sigma}}\right]\right] .
$$

In summary, the effective mean-free-path model proposed above can be described as follows. The two $g_{\mathbf{v}}^{\sigma}(\varepsilon, \mathbf{r})$ in Eq. (12) and $f_{\mathbf{v}}^{\sigma}(\varepsilon, \mathbf{r})$ in Eq. (13) are related to $\lambda_{e f f, \mathbf{v}}^{\sigma}(\mathbf{r}),{ }^{18}$ and then $\lambda_{\text {eff, },}^{\sigma}(\mathbf{r})$ in Eq. (22) is related to $D_{e q u, \mathbf{v}}^{\sigma}$. The only unde-

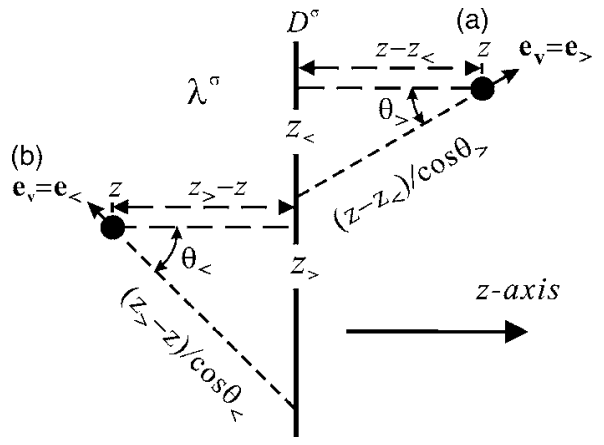

FIG. 3. Oblique transport with angles (a) $\theta_{>}$and (b) $\theta_{<}$for the right and left transport directions, respectively. The diffusive boundary specified by $D^{\sigma}$ are at $z_{<}$and $z_{>}$for the right-transport and the left-transport electrons, respectively. The solid circle represents the transport electron at $z$, and the solid arrow indicates the direction of the transport electron. The spin-dependent mean free path is $\lambda^{\sigma}$.

termined $D_{e q u, \mathbf{v}}^{\sigma}$ can be determined by use of the diagrammatical method as well as the effective diffusive scattering specified by Eq. (16) and the traveling factors $t_{\mathrm{v}}^{\sigma}$ in Eq. (17).

\section{EQUIVALENCE IN MULTILAYERED STRUCTURES WITH REALISTIC BOUNDARY CONDITIONS}

In a layered structure shown as Fig. 3, $\left|\mathbf{r}-\mathbf{r}_{<(>)}\right|$can be written as

$$
\begin{gathered}
\left|\mathbf{r}-\mathbf{r}_{<}\right|=\frac{z-z_{<}}{\cos \theta_{>}}, \\
\left|\mathbf{r}-\mathbf{r}_{>}\right|=\frac{z_{>}-z}{\cos \theta_{<}} .
\end{gathered}
$$

Substituting Eqs. (23) and (24) into Eq. (22) yields the deviation distribution $g_{>(<)}^{\sigma}(\varepsilon, z)$ by use of Eqs. (12), which is expressed by

$$
g_{>(<)}^{\sigma}(\varepsilon, z)=\frac{\partial f^{0, \sigma}(\varepsilon)}{\partial \varepsilon} \mathbf{e}_{\mathbf{v}} \cdot e \mathbf{E} \lambda^{\sigma}\left[1-D_{e q u,>(<)}^{\sigma} \exp \left[\frac{\mp\left(z-z_{<(>)}\right) / \cos \theta_{>(<)}}{\lambda^{\sigma}}\right]\right] .
$$

The $g_{>(<)}^{\sigma}(\varepsilon, z)$ in Eq. (25) has the same form of the general solution obtained from the Boltzmann transport equation model according to Eqs. (2) and (7). In terms of $g_{>(<)}^{\sigma}(\varepsilon, z)$, the only difference is between the equilibrium parameters $D_{e q u,>(<)}^{\sigma}$ in the effective mean-free-path model and the undetermined coefficients $G_{>(<)}^{\sigma}$ in the Boltzmann transport equation model. If all $D_{e q u,>(<)}^{\sigma}$ satisfy the boundary equations (8)-(11), $D_{e q u,>(<)}^{\sigma}$ are equivalent to $G_{>(<)}^{\sigma}$. Then, the equivalence between the two models is proven. In the subsequent subsections, the analytic solution for $D_{e q u, \mathbf{v}}^{\sigma}$ will be directly written down by use of the diagrammatical method in the effective mean-free-path model. The equivalent relation $D_{e q u, \mathbf{v}}^{\sigma}=G_{\mathbf{v}}^{\sigma}$ will be verified for a trilayer with simple boundary conditions ${ }^{4}$ in Sec. IV A and with complicated boundary conditions in Sec. IV B and for the multilayer structures with realistic boundary conditions in Sec. IV C.

\section{A. Trilayer structures with simple boundary conditions}

In an $A-B-C$ trilayer structure, the $A$ layer and the $C$ layer are separated by the $B$ layer with thickness $a, c$, and $b$, re- 


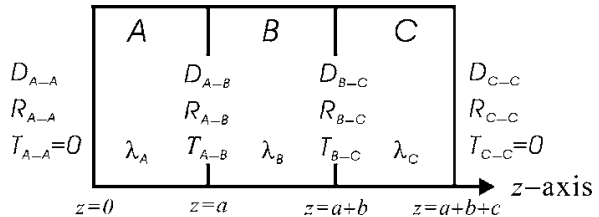

FIG. 4. $A-B-C$ trilayer structure. The diffusion parameter $D$, the nondiffusive reflection parameter $R$, the nondiffusive transmission parameter $T$, the mean free path $\lambda$, the thickness $a, b$ and $c$, and the $z$ axis are shown.

spectively (as depicted in Fig. 4). Throughout this paper, we denote all physical variables by the subscripts $A(B, C), A$ - $B(B-C)$, and $A-A(C-C)$ in order to indicate the layer, the interface, and the outer surface, respectively. The $A-A$ surface, the $A-B$ interface, the $B-C$ interface, and the $C-C$ surface are assigned to be at $z=0, z=a, z=a+b$, and $z=a+b$ $+c$, respectively. For simplicity, $A, B$, and $C$ are further used to represent the transport electron both inside the corresponding layer and with the corresponding spin state; for example, $\lambda_{A}$ represents $\lambda_{A}^{\sigma}$, which is the spin- $\sigma$ mean free path at the $A$ layer. For the $A-B-C$ trilayer structure, the undetermined coefficients in Eq. (7) are transformed into $G_{A,>(<)}, G_{B,>(<)}$, and $G_{C,>(<)}$; also, the equilibrium parameters in Eq. (25) are transformed into $D_{e q u, A,>(<)}, D_{e q u, B,>(<)}$, and $D_{e q u, C,>(<)}$.

In the $A-B-C$ trilayer structure, the simple boundary conditions are given as follows: ${ }^{4}$ (a) $T_{A-A}=T_{C-C}=0$; (b) $R_{A-B}$ $=R_{B-C}=0$; (c) the reflected and the transmitted angles are the same as the incident angle $\theta_{i n}=\theta$ for the transport electron, and (d) the same spin-independent mean free paths are $\lambda_{A}$ $=\lambda_{B}=\lambda_{C}=\lambda$. Given these simple boundary conditions, the solutions for $D_{e q u, A,>(<)}, D_{e q u, B,>(<)}$, and $D_{e q u, C,>(<)}$ can be systematically written down by use of the diagrammatical method as well as the effective diffusive scattering specified by Eq. (16) and the traveling factors $t_{\mathbf{v}}^{\sigma}$ in Eq. (17).

For example, $D_{e q u, A,>}$ on the unit right-transport electron at $z=0$ inside the $A$ layer can be directly written down. The value of $D_{e q u, A,>}$ is equivalent to the determination to specify all effective diffusive scatterings on an injected unit lefttransport electron from $z=0$. All individual effective diffusive scatterings on this incident left-transport electron depend on their related transport paths, and are analyzed stepwise as follows (Fig. 5). (a) The injected unit left-

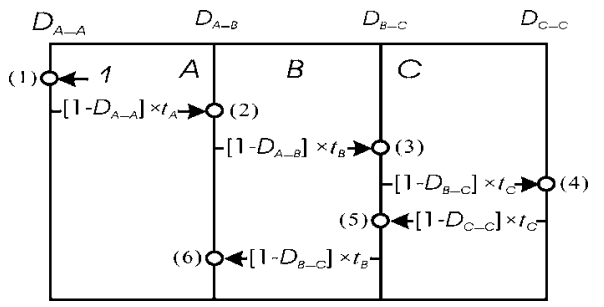

FIG. 5. Transport diagram for the determination of $D_{e q u, A,>}$ in the $A-B-C$ trilayer structure. Open circles represent the effective diffusive scatterings, specified by (1) $D_{1}$, (2) $D_{2}$, (3) $D_{3}$, (4) $D_{4}$, (5) $D_{5}$, and (6) $D_{6}$, on the transport electron; $t$ is the traveling factor. Arrows indicate the direction of the transport electron to travel. transport electron from $z=0$ is first scattered at the diffusive $A-A$ surface with $D_{A-A}$, and thus this electron undergoes the first effective diffusive scattering, which is specified by $D_{1}$ $=1 \times D_{A-A}$. This transport path from the left to the right side of $A-A$ surface is the first one. (b) Next, the electron flux of the residual transport electron that has traveled along the first transport path is $\left[1-D_{A-A}\right]$, and is reflected by the $A-A$ surface, then traveling a distance $a$ and being scattered at the diffusive $A-B$ interface with $D_{A-B}$. This transport path, the second transport path is from the $A-A$ surface at $z=0$ to the $A-B$ interface at $z=a$, and contains the traveling factor $t_{A}$ $=\exp [-a / \lambda \cos \theta]$ for the transport electron in $A$ layer. Hence, the effective diffusive scattering associated with the second transport path can be specified by $D_{2}=\left[1-D_{A-A}\right]$ $\times t_{A} \times D_{A-B}$, which is on the unit injected left-transport electron from $z=0$. (c) The electron flux of the residual transport electron that has traveled along the second transport path is $\left[1-D_{A-A}\right] \times t_{A} \times\left[1-D_{A-B}\right]$ and is transmitted by the $A-B$ interface. Then this electron travels a distance $b$ and is scattered at the diffusive $B-C$ interface, in which the corresponding traveling factor is $t_{B}=\exp [-b / \lambda \cos \theta]$ for this transport electron in $B$ layer and the diffusive $B-C$ interface is specified by $D_{B-C}$. This transport path, the third transport path is from the $A-A$ surface to the $A-B$ interface to the $B$ - $C$ interface. Therefore, the effective diffusive scattering associated with the third transport path can be specified by $D_{3}=[1$ $\left.-D_{A-A}\right] \times t_{A} \times\left[1-D_{A-B}\right] \times t_{B} \times D_{B-C}$. (d) Likewise, the corresponding effective diffusive scatterings associated with the fourth, the fifth, and the sixth transport paths are specified by $D_{4}, D_{5}$, and $D_{6}$, respectively. These $D_{4}, D_{5}$, and $D_{6}$ can be directly and easily written down below. The mathematical structure for the determination of $D_{1}, D_{2}, D_{3}, D_{4}, D_{5}$, and $D_{6}$ in $D_{e q u, A,>}$ is shown as the transport diagram in Fig. 5. These $D_{1}, D_{2}, D_{3}, D_{4}, D_{5}$ and $D_{6}$ are

$$
\begin{gathered}
D_{1}=D_{A-A}, \\
D_{2}=\left[1-D_{A-A}\right] D_{A-B} \exp \left[\frac{-a}{\lambda \cos \theta}\right], \\
D_{3}=\left[1-D_{A-A}\right]\left[1-D_{A-B}\right] D_{B-C} \exp \left[\frac{-a-b}{\lambda \cos \theta}\right], \\
D_{4}=\left[1-D_{A-A}\right]\left[1-D_{A-B}\right]\left[1-D_{B-C}\right] D_{C-C} \exp \left[\frac{-a-b-c}{\lambda \cos \theta}\right], \\
D_{5}=\left[1-D_{A-A}\right]\left[1-D_{A-B}\right]\left[1-D_{B-C}\right]\left[1-D_{C-C}\right] D_{B-C} \\
\times \exp \left[\frac{-a-b-2 c}{\lambda \cos \theta}\right], \\
D_{6}=\left[1-D_{A-A}\right]\left[1-D_{A-B}\right]\left[1-D_{B-C}\right]\left[1-D_{C-C}\right]\left[1-D_{B-C}\right] \\
\times D_{A-B} \exp \left[\frac{-a-2 b-2 c}{\lambda \cos \theta}\right] .
\end{gathered}
$$

(e) The electron flux of the residual transport electron that 
has traveled the sixth transport path is transmitted by the $A$ $-B$ interface, and then travels a distance $a$ and is scattered at the diffusive $A-A$ surface again. Hence, the effective diffusive scattering associated with the seventh transport path is specified by $D_{7}=L \times D_{1}$, where

$$
\begin{aligned}
L= & {\left[1-D_{A-A}\right]\left[1-D_{A-B}\right]\left[1-D_{B-C}\right]\left[1-D_{C-C}\right]\left[1-D_{B-C}\right] } \\
& \times\left[1-D_{A-B}\right] \exp \left[\frac{-2 a-2 b-2 c}{\lambda \cos \theta}\right] .
\end{aligned}
$$

The loop factor $L$ for the $A-B-C$ trilayer in Eq. (32) represents the electron flux ratio of the residual to the initial incident transport electron after it has traveled along a single and close loop within the $A-B-C$ trilayer. This single and close loop is the transport path from the $A-A$ surface to the $A-B$ interface, to the $B$ - $C$ interface, to the $C$ - $C$ surface, to the $B$ - $C$ interface, to the $A-B$ interface, and back to the $A-A$ surface. Furthermore, it is straightforward that $D_{6 n+i}=D_{i} \times L^{n}$ for $i=1,2,3,4,5,6$ and $n=0,1,2 \ldots$. Therefore, the superposition of the determinations to specify all effective diffusive scatterings, $D_{\text {equ }, A,>}$ is

$$
D_{e q u, A,>}=\frac{D_{1}+D_{2}+D_{3}+D_{4}+D_{5}+D_{6}}{1-L} .
$$

Similarly, $D_{e q u, B,>}$ on the unit right-transport electron at $z=a$ can be directly written down by use of the diagrammatical method. Inject a unit left-transport electron from $z=a$ into the $A-B-C$ trilayer. The injected electron is first scattered at the diffusive $A-B$ interface with $D_{A-B}$, and then the residual transport electron with the electron flux of $\left[1-D_{A-B}\right]$ travels a distance $a$ and is scattered at the $A-A$ diffusive surface, then repeating all processes in the determination of $D_{e q u, A,>}$, such that

$$
D_{e q u, B,>}=D_{A-B}+\left[1-D_{A-B}\right] \exp \left[\frac{-a}{\lambda \cos \theta}\right] \times D_{e q u, A,>} .
$$

Substituting Eqs. (33) and (34) into Eq. (25) can easily be verified to satisfy the boundary equation, Eq. (8) with $R_{A-B}$ $=0$. Similarly, all equilibrium diffusion parameters can be written down, and indeed satisfy the simple boundary equations. Accordingly, $D_{e q u}^{\sigma}=G^{\sigma}$ holds for the trilayer structure with simple boundary conditions.

\section{B. Trilayer structures with more general boundary conditions}

More general boundary conditions that $\lambda_{A}^{\sigma} \neq \lambda_{B}^{\sigma} \neq \lambda_{C}^{\sigma}$ and $R_{B-C} \neq 0$ can be considered for the $A-B-C$ trilayer structure. In this subsection, we first investigate the $A-B-C$ trilayer structure with $\lambda_{A} \neq \lambda_{B} \neq \lambda_{C}$ and then with the combined $R_{B-C} \neq 0$ and $\lambda_{A} \neq \lambda_{B} \neq \lambda_{C}$.

When the $A-B-C$ trilayer structure is with $\lambda_{A} \neq \lambda_{B} \neq \lambda_{C}$ instead of $\lambda_{A}=\lambda_{B}=\lambda_{C}=\lambda$, the effective diffusive scatterings on the transport electron will be modified accordingly. To understand the modified effective diffusive scatterings due to the influence of different mean free paths between adjacent materials, we study an $A-B$ bilayer with two infinite planes, where the positions of the boundaries are assigned $z_{A,>}=0$,

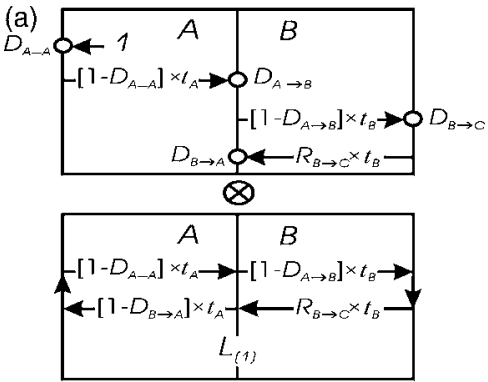

(b)

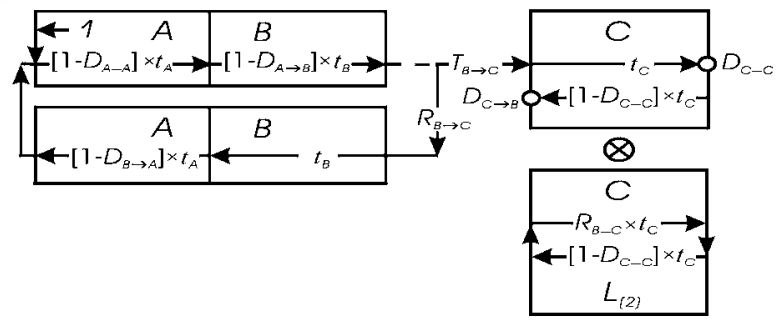

(c)

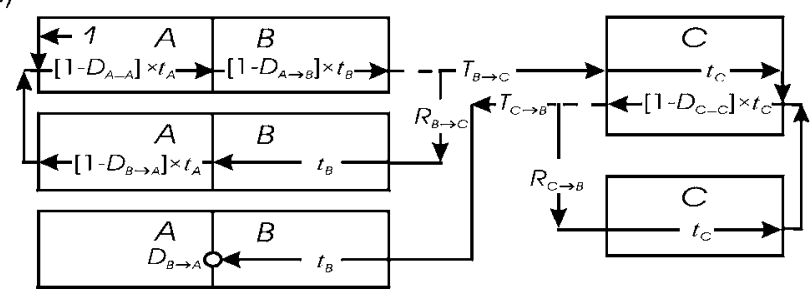

FIG. 6. Transport diagram for the determination of $D_{\text {equ,A, }}$ in the $A-B-C$ trilayer structure with the reflective $B-C$ interface and with the different mean free paths $\lambda_{A} \neq \lambda_{B} \neq \lambda_{C}$. Three inner loops (a), (b) and (c) traveled by the incident transport electron are diagrammatically shown. Open circles represent the effective diffusive scatterings on the transport electron. Arrows indicate the directions of the electron transport. At the $B-C$ interface, the transport electron along the dashed line can be reflected by $R_{B \rightarrow C}\left(R_{C \rightarrow B}\right)$ or transmitted by $T_{B \rightarrow C}\left(T_{C \rightarrow B}\right)$. The loop factors $L_{(1)}$ and $L_{(2)}$ for the $A-B$ bilayer and the $C$ layer are diagrammatically shown in (a) and (b), respectively. The symbol $\otimes$ indicates the determination of the effective diffusive scatterings on the transport electron multiplied by $L_{(1)}$ and $L_{(2)}$. The $D$ is the modified diffusion parameter, and $t$ is the traveling factor.

$z_{A,<}=-\infty$ and $z_{B,>}=+\infty, z_{B,<}=0$. Meanwhile, we define the modified diffusion parameter $D_{A \rightarrow B}\left(D_{B \rightarrow A}\right)$ to specify the diffusive scattering from the $A-B$ interface on a transport electron at the $A-B$ interface when this electron tends to transport along the direction from the $A(B)$ layer through the $A-B$ interface to the $B(A)$ layer. In the $A-B$ bilayer, these two $D_{A \rightarrow B}$ and $D_{B \rightarrow A}$ at the $A-B$ interface are equal to $D_{e q u, A,<}$ and $D_{e q u, B,>}$, respectively, because the effective diffusive scatterings from the boundaries $z_{A,<}=-\infty$ and $z_{B,>}=+\infty$ can be ignored according to the effective mean-free-path model. That is to say, $D_{A \rightarrow B}=D_{e q u, A,<}$ and $D_{B \rightarrow A}=D_{e q u, B,>}$ in this $A-B$ bilayer. Besides, $D_{e q u, A,<}=G_{A,<}$ and $D_{e q u, B,>}=G_{B,>}$ if the equivalence must hold between the effective mean-freepath model and the Boltzmann transport equation model. Therefore, $\quad D_{A \rightarrow B}=D_{e q u, A,<}=G_{A,<} \quad$ and $\quad D_{B \rightarrow A}=D_{e q u, B,>}$ $=G_{B,>}$. Then, the modified diffusion parameters $D_{A \rightarrow B}$ and $D_{B \rightarrow A}$ can be given after algebraic manipulation of the Bolt- 
zmann transport equation model yields $G_{A,<}$ and $G_{B,>}$, where

$$
\begin{gathered}
G_{A,<}=1-R_{A-B}-\frac{\lambda_{B}}{\lambda_{A}}\left[1-D_{A-B}\right], \\
G_{B,>}=1-R_{A-B}-\frac{\lambda_{A}}{\lambda_{B}}\left[1-D_{A-B}\right] .
\end{gathered}
$$

Hence, $D_{A \rightarrow B}$ and $D_{B \rightarrow A}$ are given by

$$
\begin{aligned}
& D_{A \rightarrow B}=1-R_{A \rightarrow B}-T_{A \rightarrow B}, \\
& D_{B \rightarrow A}=1-R_{B \rightarrow A}-T_{B \rightarrow A},
\end{aligned}
$$

where the modified reflection parameters are $R_{A \rightarrow B}=R_{A-B}$ and $R_{B \rightarrow A}=R_{B-A}$, and the modified transmission parameters are $T_{A \rightarrow B}=\lambda_{B} / \lambda_{A} \times T_{A-B}$ and $T_{B \rightarrow A}=\lambda_{A} / \lambda_{B} \times T_{A-B}$.

Equations (35)-(38) indicate that the asymmetry of $\lambda_{B} / \lambda_{A} \neq \lambda_{A} / \lambda_{B}$ causes $D_{A \rightarrow B} \neq D_{B \rightarrow A} \neq D_{A-B}$. The two $D_{A \rightarrow B}$ and $D_{B \rightarrow A}$ return to the interfacial $D_{A-B}$ when $\lambda_{A}=\lambda_{B}$. It should be noted that even given $D_{A-B}=0, D_{A \rightarrow B}\left(D_{B \rightarrow A}\right)$ still exists when $\lambda_{A} \neq \lambda_{B}$. This implies that the asymmetry of $\lambda_{A} / \lambda_{B} \neq \lambda_{B} / \lambda_{A}$ induces a directional diffusive scattering; therefore, no perfect interface exists when there is the mismatch between the transport properties. Moreover, when $\lambda_{A}$ or $\lambda_{B}$ or both are spin-dependent, the induced diffusive scattering at the interface becomes not only directional but also spin-dependent. For example, an induced directional spindependent interfacial scattering occurs at the ferromagnetmetal interface due to the asymmetry in the mean free path between a bulk ferromagnet and a bulk metal. In terms of the effective mean-free-path model, this induced interfacial scattering has a maximum when the transport direction of the electrons is perpendicular to the interface and vanishes when the direction is parallel to the interface. Hence, this induced interfacial scattering is more important for the CPP (current perpendicular to plane) geometry than for the CIP (current parallel to plane) geometry of a magnetic multilayered structure.

After the modified diffusion parameters are introduced to include the influence of the asymmetry in the different mean free paths between adjacent materials, the equilibrium parameter $D_{e q u, A,>(<)}, D_{e q u, B,>(<)}$, and $D_{e q u, C,>(<)}$ for the $A-B$ $-C$ trilayer structure with the combined $R_{B-C} \neq 0$ and $\lambda_{A}$ $\neq \lambda_{B} \neq \lambda_{C}$ can be determined. Take $D_{\text {equ,A,> }}$ as an example. The value of $D_{e q u, A,>}$ is equivalent to the determination to specify all effective diffusive scatterings on the injected unit left-transport electron from $z=0$. Due to an additional reflective $B-C$ interface in the $A-B-C$ trilayer structure, all effective diffusive scatterings on this injected electron come from not only one simple loop. Indeed, all transport paths can be categorized into three inner loops as well as one outer loop (Fig. 6). Then, $D_{e q u, A,>}$ for the $A-B-C$ trilayer structure with the combined $R_{B-C} \neq 0$ and $\lambda_{A} \neq \lambda_{B} \neq \lambda_{C}$ is given by

$$
\begin{aligned}
& D_{e q u, A,>}=\frac{\frac{1}{1-L_{(1)}} \times D_{(1)}+\frac{t_{(1)}}{1-L_{(1)}} \times \frac{1}{1-L_{(2)}} \times D_{(2)}+\frac{t_{(1)}}{1-L_{(1)}} \times \frac{t_{(2)}}{1-L_{(2)}} \times D_{(3)}}{1-L_{(3)}}, \\
& D_{(1)}=+D_{A-A}+\left[1-D_{A-A}\right] D_{A \rightarrow B} \exp \left[\frac{-a}{\lambda_{A} \cos \theta}\right] \\
& +\left[1-D_{A-A}\right]\left[1-D_{A \rightarrow B}\right] D_{B \rightarrow C} \\
& \times \exp \left[\frac{-a}{\lambda_{A} \cos \theta}\right] \exp \left[\frac{-b}{\lambda_{B} \cos \theta}\right] \\
& +\left[1-D_{A-A}\right]\left[1-D_{A \rightarrow B}\right] R_{B \rightarrow C} D_{B \rightarrow A} \\
& \times \exp \left[\frac{-a}{\lambda_{A} \cos \theta}\right] \exp \left[\frac{-2 b}{\lambda_{B} \cos \theta}\right] \text {, } \\
& D_{(2)}=+D_{C-C} \exp \left[\frac{-c}{\lambda_{C} \cos \theta}\right] \\
& +\left[1-D_{C-C}\right] D_{C \rightarrow B} \exp \left[\frac{-2 c}{\lambda_{C} \cos \theta}\right] \text {, } \\
& D_{(3)}=D_{B \rightarrow A} \exp \left[\frac{-b}{\lambda_{B} \cos \theta}\right] \text {, } \\
& L_{(1)}=\left[1-D_{A-A}\right]\left[1-D_{A \rightarrow B}\right] R_{B \rightarrow C}\left[1-D_{B \rightarrow A}\right] \\
& \times \exp \left[\frac{-2 a}{\lambda_{A} \cos \theta}\right] \exp \left[\frac{-2 b}{\lambda_{B} \cos \theta}\right] \text {, } \\
& L_{(2)}=\left[1-D_{C-C}\right] R_{C \rightarrow B} \exp \left[\frac{-2 c}{\lambda_{C} \cos \theta}\right] \text {, } \\
& L_{(3)}=\frac{t_{(1)}}{1-L_{(1)}} \times \frac{t_{(2)}}{1-L_{(2)}} \times t_{(3)}, \\
& t_{(1)}=\left[1-D_{A-A}\right]\left[1-D_{A \rightarrow B}\right] T_{B \rightarrow C} \exp \left[\frac{-a}{\lambda_{A} \cos \theta}\right] \\
& \times \exp \left[\frac{-b}{\lambda_{B} \cos \theta}\right] \text {, } \\
& t_{(2)}=\left[1-D_{C-C}\right] T_{C \rightarrow B} \exp \left[\frac{-2 c}{\lambda_{C} \cos \theta}\right] \text {, }
\end{aligned}
$$




$$
t_{(3)}=\left[1-D_{B \rightarrow A}\right] \exp \left[\frac{-a}{\lambda_{A} \cos \theta}\right] \exp \left[\frac{-b}{\lambda_{B} \cos \theta}\right] .
$$

Without the reflective $B-C$ interface and with $\lambda_{A}=\lambda_{B}=\lambda_{C}$, Eq. (39) indeed reduces to Eq. (33). It should be noted that $D_{A \rightarrow B} \neq D_{B \rightarrow A} \neq D_{A-B}$ and $D_{B \rightarrow C} \neq D_{C \rightarrow B} \neq D_{B-C}$ due to $\lambda_{A}$ $\neq \lambda_{B} \neq \lambda_{C}$. Similarly, other equilibrium parameters $D_{\text {equ }, A,<}$, $D_{e q u, B,>(<)}$, and $D_{e q u, C,>(<)}$ in this $A-B-C$ trilayer structure can be obtained. It can be verified easily that all equilibrium parameters obtained from the effective mean-free-path model satisfy the boundary equations, Eqs. (8)-(11). Hence, $D_{\text {equ }}^{\sigma}$ $=G^{\sigma}$ still holds in the trilayer structure with more general boundary conditions that $\lambda_{A} \neq \lambda_{B} \neq \lambda_{C}$ and $R_{B-C} \neq 0$.

\section{Multilayered structures with realistic boundary conditions}

In this subsection, the equivalence between the effective mean-free-path model and the Boltzmann transport equation model will be generalized to multilayered structures with realistic boundary conditions. The realistic boundary conditions contain numerous reflective interfaces, different mean free paths between adjacent materials, and the relations among the incident angle $\theta_{i n}$, the transmitted angle $\theta_{t}$, and the reflected angle $\theta_{r}$.

First, this equivalence can be generalized to multilayered structures with numerous reflective interfaces. Studies of trilayer structures without and with a reflective interface reveal that the role of the reflective interface in the effective mean-free-path model plays a beam-splitting role for the transport electron beam. The presence of the reflective interface just causes the transport electron to be not only transmitted but also reflected. Both in the presence and in the absence of reflective interfaces, the effective mean-free-path model is equivalent to the Boltzmann transport equation model. Therefore, this equivalence holds for multilayered structures with numerous reflective interfaces.

Second, this equivalence can be generalized to multilayered structures. A multilayered structure consists of different materials. Their transport properties can be completely specified by their corresponding mean free paths in terms of the Boltzmann transport equation model, and also the effective mean-free-path model. So, the mismatch between the transport properties can be specified by the asymmetry in the different mean free paths between adjacent materials. In comparison with the studies on the $A-B-C$ trilayer with $\lambda_{A}$ $\neq \lambda_{B} \neq \lambda_{C}$ and with $\lambda_{A}=\lambda_{B}=\lambda_{C}$, it reveals that this mismatch induces a diffusive scattering and modifies the interfacial diffusion parameter as the directional modified diffusion parameter. By use of the modified diffusion parameters instead of the interfacial diffusion parameters, this equivalence can be generalized to multilayered structures.

Third, this equivalence can be generalized to multilayered structures with $\theta_{\text {in }} \neq \theta_{r} \neq \theta_{t}$. The difference between $\theta_{\text {in }} \neq \theta_{r}$ $\neq \theta_{t}$ and $\theta_{i n}=\theta_{r}=\theta_{t}$ affects the corresponding traveling distance, and then modifies the traveling factor $t_{\mathbf{v}}^{\sigma}$ in Eq. (17) in its magnitude. This does not change the formalism of the effective mean-free-path model; both the schematic structure of the transport diagrams such as Fig. 5 and the mathematical structure for answers such as Eq. (33) still hold. Hence this equivalence can be generalized to multilayered structures with $\theta_{\text {in }} \neq \theta_{r} \neq \theta_{t}$ by use of extra considerations to the magnitude of the corresponding traveling factor $t_{\mathbf{v}}^{\sigma}$.

Therefore, the equivalence between the effective meanfree-path model and the Boltzmann transport equation model is generalized to multilayered structures with realistic boundary conditions. The equivalent solution for the electron distribution to the Boltzmann transport equation model can be written down according to the effective mean-free-path model. Given the boundary conditions, even if they are very sophisticated, the effective mean-free-path model can be easily used to determine the electron distribution.

\section{EQUIVALENCE IN MULTILAYERED STRUCTURES WITH VARIOUS SHAPES}

The effective mean-free-path model can be applied to find the general solutions for the electron distributions $f_{\mathbf{v}}^{\sigma}(\varepsilon, \mathbf{r})$ in multilayered structures with various shapes. First, the general solution for $f_{\mathbf{v}}^{\sigma}(\varepsilon, \mathbf{r})$ depends on the general solution for the boundary chemical potential $\Delta \mu_{\mathbf{v}}^{\sigma}(\mathbf{r})$ according to Eqs. (2) and (5). Next, the general solution for $f_{\mathbf{v}}^{\sigma}(\varepsilon, \mathbf{r})$ depends on the effective mean free path $\lambda_{\text {eff, } \mathbf{v}}^{\sigma}(\mathbf{r})$ in Eq. (22) according to Eqs. (13). Due to the same $f_{\mathbf{v}}^{\sigma}(\varepsilon, \mathbf{r})$, there exists a relation between $\Delta \mu_{\mathbf{v}}^{\sigma}(\mathbf{r})$ and $\lambda_{e f f, \mathbf{v}}^{\sigma}(\mathbf{r})$, which is

$$
\Delta \mu_{>(<)}^{\sigma}(\mathbf{r})=\mathbf{e}_{>(<)} \cdot e \mathbf{E} \lambda^{\sigma} D_{e q u,>(<)}^{\sigma} \times \exp \left[\frac{-\left|\mathbf{r}-\mathbf{r}_{<(>)}\right|}{\lambda^{\sigma}}\right] .
$$

Now, the general solution for $\Delta \mu_{\mathbf{v}}^{\sigma}(\mathbf{r})$ is the algebraic expression in Eq. (49) based on the effective mean-free-path model. It can be easily written down, instead of hard finding the general solution from the differential equation in Eq. (6) in terms of the Boltzmann transport equation model. Equation (49) indicates a requirement for the transport electron that $\Delta \mu_{\mathbf{v}}^{\sigma}(\mathbf{r})$ related to $\lambda_{\text {eff, }}^{\sigma}(\mathbf{r})$ decays exponentially as the ratio of the distance from the diffusive source to the mean free path $\lambda^{\sigma}$ increases. According to the requirement, the conventional general solutions for $f_{\mathbf{v}}^{\sigma}(\varepsilon, \mathbf{r})$ in structures with various shapes can be easily obtained. Therefore, the equivalence between the effective mean-free-path model and the Boltzmann transport equation model can be generalized to multilayered structures with various shapes. Two examples illustrate the determination for $\Delta \mu_{\mathbf{v}}^{\sigma}(\mathbf{r})$ according to Eq. (49). One is the general solution $\Delta \mu_{\mathbf{v}}^{\sigma}(\mathbf{r})$ for a solid sphere in Sec. $\mathrm{V} \mathrm{A}$, and another is for an infinite thin cylindrical wire in Sec. V B.

\section{A. Solid sphere}

In the solid sphere depicted in Fig. 7, the transport electron with the velocity $v_{F} \mathbf{e}_{\mathbf{v}}$ is at the position $\mathbf{r}$. With respect to the center of the solid sphere, the origin $O_{\mathbf{r}}$, the position of the transport electron is defined as $\mathbf{r}=\left(r, \theta_{\mathbf{r}}, \phi_{\mathbf{r}}\right)$ in spherical coordinates. The extended lines from $\mathbf{e}_{\mathbf{v}}$ and from $-\mathbf{e}_{\mathbf{v}}$ intersecting the sphere at $r=r_{0}$ are points $O_{D 1}$ and $O_{\mathbf{v}}$, respectively, where $r_{0}$ is the radius of the solid sphere. At the origin $O_{\mathbf{v}}, \mathbf{e}_{\mathbf{v}}$ is defined as $\mathbf{e}_{\mathbf{v}}=\mathbf{e}_{\mathbf{v}}\left(\theta_{\mathbf{v}}, \phi_{\mathbf{v}}\right)$ in spherical coordinates. 


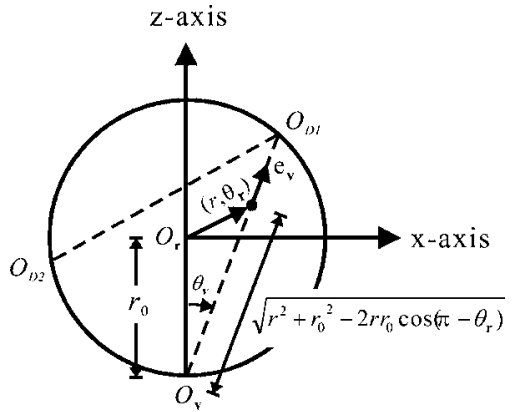

FIG. 7. Solid sphere with the radius $r_{0}$ in the $x-z$ plane. The solid circle represents the transport electron at $\mathbf{r}=\left(r, \theta_{\mathbf{r}}\right)$, and the direction of the transport electron is $\mathbf{e}_{\mathbf{v}}=\mathbf{e}_{\mathbf{v}}\left(\theta_{\mathbf{v}}, \phi_{\mathbf{v}}=0\right)$. The electron reflected at $O_{D 1}$ travels to the point $O_{D 2}$, where $\overline{O_{\mathbf{v}} O_{D 1}}=\overline{O_{D 1} O_{D 2}}$ $=2 r_{0} \cos \theta_{>(<)}$according to the electron reflection law.

The line that connects $O_{\mathbf{y}}$ to $O_{\mathbf{r}}$ is directed along the $z$ axis, where the distance, $\overline{O_{\mathbf{v}} O_{\mathbf{r}}}$, is $r_{0}$. The relation between $\mathbf{e}_{\mathbf{r}}$ and $\mathbf{e}_{\mathbf{v}}$ is

$$
\mathbf{e}_{\mathbf{v}}=\cos \left(\theta_{\mathbf{r}}-\theta_{\mathbf{v}}\right) \mathbf{e}_{\mathbf{r}}-\sin \left(\theta_{\mathbf{r}}-\theta_{\mathbf{v}}\right) \mathbf{e}_{\theta_{\mathbf{r}}},
$$

and then Eq. (6) in the Boltzmann transport equation model becomes

$$
\cos \left(\theta_{\mathbf{r}}-\theta_{\mathbf{v}}\right) \frac{\partial[\Delta \mu]}{\partial r}-\sin \left(\theta_{\mathbf{r}}-\theta_{\mathbf{v}}\right) \frac{1}{r} \frac{\partial[\Delta \mu]}{\partial \theta_{\mathbf{r}}}=-\frac{[\Delta \mu]}{\lambda}
$$

Here, $\mathbf{v}$ is also divided into $>(<)$ to represent to the transport direction of the electron to the forward (backward) boundary at $O_{D 1}\left(O_{\mathbf{v}}\right)$. Accordingly, $\theta_{>}$is defined as $0 \leqslant \theta_{\mathbf{v}}$ $\leqslant \pi / 2$ to represent the transport direction away the point $O_{\mathrm{v}}$, and $\theta_{<}=\pi-\theta_{\mathbf{v}}$ is defined as $\pi / 2 \leqslant \theta_{\mathbf{v}} \leqslant \pi$ to represent the transport direction to the point $O_{\mathbf{v}}$.

According to Eq. (49) in the effective mean-free-path model, the boundary chemical potential $\Delta \mu_{>(<)}^{\sigma}\left(r, \theta_{\mathbf{r}}\right)$ is

$\Delta \mu_{>(<)}^{\sigma}\left(r, \theta_{\mathbf{r}}\right)=\mathbf{e}_{>(<)} \cdot e \mathbf{E} \lambda^{\sigma} D_{e q u,>(<)}^{\sigma} \times \exp \left[\frac{-\left|\mathbf{r}-\mathbf{r}_{<(>)}\right|}{\lambda^{\sigma}}\right]$.

In the solid sphere shown in Fig. 7, the distance of $\left|\mathbf{r}-\mathbf{r}_{>}\right|$ between the transport electron and the forward boundary at $O_{D 1}$ is

$$
\left|\mathbf{r}-\mathbf{r}_{>}\right|=2 r_{0} \cos \theta_{>}-\sqrt{r^{2}+r_{0}^{2}-2 r r_{0} \cos \left(\pi-\theta_{\mathbf{r}}\right)},
$$

and the distance of $\left|\mathbf{r}-\mathbf{r}_{<}\right|$between the transport electron and the backward boundary at $O_{\mathbf{v}}$ is

$$
\left|\mathbf{r}-\mathbf{r}_{<}\right|=\sqrt{r^{2}+r_{0}^{2}-2 r r_{0} \cos \left(\pi-\theta_{\mathbf{r}}\right)} .
$$

Then, the boundary chemical potential $\Delta \mu_{>(<)}^{\sigma}\left(r, \theta_{\mathbf{r}}\right)$ can be written as

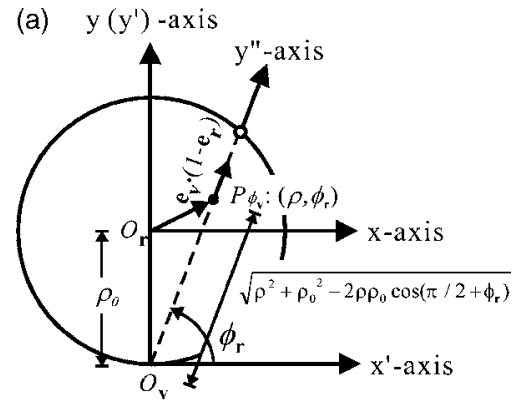

(b)

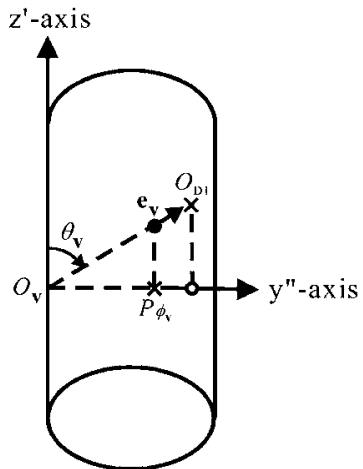

FIG. 8. Cylindrical wire with the radius $\rho_{0}$ in (a) the $x-y\left(x^{\prime}\right.$ $\left.-y^{\prime}\right)$ plane and (b) in the $y^{\prime \prime}-z^{\prime}$ plane. The $y^{\prime \prime}-z^{\prime}$ plane is the plane $y^{\prime}-z^{\prime}$ rotated counterclockwise through an angle of $\pi / 2-\phi_{\mathbf{v}}$ about the $z^{\prime}$ axis. The transport electron with $\mathbf{e}_{\mathbf{v}}=\mathbf{e}_{\mathbf{v}}\left(\theta_{\mathbf{v}}, \phi_{\mathbf{v}}\right)$ at $\mathbf{r}$ $=\left(\rho, \phi_{\mathbf{r}}, z\right)$ travels away from the point $O_{\mathbf{v}}$ and toward the point $O_{D 1}$. The point $P_{\phi_{\mathbf{v}}}$ is at $\left(\rho, \phi_{\mathbf{r}}, z=0\right)$.

$$
\begin{aligned}
\Delta \mu_{>(<)}^{\sigma}\left(r, \theta_{\mathbf{r}}\right)= & \mathbf{e}_{>(<)} \cdot e \mathbf{E} \lambda^{\sigma} D_{e q u,>(<)}^{\sigma} \\
& \times \exp \left[\frac{\mp\left(\sqrt{r^{2}+r_{0}^{2}+2 r r_{0} \cos \theta_{\mathbf{r}}}-r_{<(>)}\right)}{\lambda^{\sigma}}\right],
\end{aligned}
$$

where $r_{<}=0$ and $r_{>}=2 r_{0} \cos \theta_{>}$. Substituting Eq. (55) into Eq. (51) easily verifies that Eq. (55) in the effective meanfree-path model is the general solution to Eq. (51) in the Boltzmann transport equation model. The only undetermined coefficient in the general solution is just the equilibrium diffusion parameter $D_{e q u,>(<)}^{\sigma}$, which depends on the given boundary conditions.

\section{B. Infinite thin cylindrical wire}

In the cylindrical wire depicted in Fig. 8 the transport electron with the velocity $v_{F} \mathbf{e}_{\mathbf{v}}$ is at the position $\mathbf{r}$. With respect to the centerline of the cylindrical wire, the position of the transport electron is defined as $\mathbf{r}=\left(\rho, \phi_{\mathbf{r}}, z\right)$ in cylindrical coordinates. The extended line from $\mathbf{e}_{\mathbf{v}}$ intersecting the surface of the cylindrical wire at $\rho=\rho_{0}$ is points $O_{\mathbf{v}}$ and $O_{D 1}$, where $\rho_{0}$ is the radius of the cylindrical wire. At the origin $O_{\mathbf{v}}, \mathbf{e}_{\mathbf{v}}$ is defined as $\mathbf{e}_{\mathbf{v}}=\mathbf{e}_{\mathbf{v}}\left(\theta_{\mathbf{v}}, \phi_{\mathbf{v}}\right)$ in spherical coordinates. Due to $z \rightarrow \pm \infty$ in an infinite cylindrical wire, the origin $O_{\mathbf{r}}$ on the $z$ axis of the infinite cylindrical can be defined by the fact that the line connecting from $O_{\mathbf{v}}$ to $O_{\mathbf{r}}$ is directed along the $y$ axis. Therefore, their distance, $\overline{O_{\mathbf{v}} O_{\mathbf{r}}}$, is $\rho_{0}$, and the 
relation between $\mathbf{e}_{\mathbf{r}}$ and $\mathbf{e}_{\mathbf{v}}$ can be expressed as

$$
\mathbf{e}_{\mathbf{v}}=\sin \theta_{\mathbf{v}} \times\left[\cos \left(\phi_{\mathbf{v}}-\phi_{\mathbf{r}}\right) \mathbf{e}_{\rho}-\sin \left(\phi_{\mathbf{v}}-\phi_{\mathbf{r}}\right) \mathbf{e}_{\phi_{\mathbf{r}}}\right]+\cos \theta_{\mathbf{v}} \mathbf{e}_{z},
$$

so Eq. (6) in the Boltzmann transport equation model becomes

$$
\begin{aligned}
& \sin \theta_{\mathbf{v}} \cos \left(\phi_{\mathbf{v}}-\phi_{\mathbf{r}}\right) \frac{\partial[\Delta \mu]}{\partial \rho}+\sin \theta_{\mathbf{v}} \sin \left(\phi_{\mathbf{v}}-\phi_{\mathbf{r}}\right) \frac{1}{\rho} \frac{\partial[\Delta \mu]}{\partial \phi}= \\
& -\frac{\Delta[\mu]}{\lambda}
\end{aligned}
$$

where $\Delta \mu\left(\rho, \phi_{\mathbf{r}}, z\right)=\Delta \mu\left(\rho, \phi_{\mathbf{r}}\right)$ for $z \rightarrow \pm \infty$ in an infinite cylindrical wire. Also, $\mathbf{v}$ is divided into $>(<)$ to represent to the transport direction of the electron to the forward (backward) boundary at $O_{D 1}\left(O_{\mathbf{v}}\right)$. Accordingly, $\phi_{>}$is defined as
$0 \leqslant \phi_{\mathbf{v}} \leqslant \pi$ to represent the transport direction away from the point $O_{\mathbf{v}}$, and $\phi_{<}=2 \pi-\phi_{\mathbf{v}}$ is defined as $\pi \leqslant \phi_{\mathbf{v}} \leqslant 2 \pi$ to represent the electron transport to the point $O_{\mathbf{v}}$.

In the cylindrical wire shown in Fig. 8, the distance of $\left|\mathbf{r}-\mathbf{r}_{>(<)}\right|$from the transport electron to the forward (backward) boundary at $O_{D 1}\left(O_{\mathbf{v}}\right)$ is

$$
\begin{gathered}
\left|\mathbf{r}-\mathbf{r}_{>}\right|=\frac{2 \rho_{0} \cos \left(\pi / 2-\phi_{>}\right)-\sqrt{\rho^{2}+\rho_{0}^{2}+2 \rho \rho_{0} \sin \phi_{\mathbf{r}}}}{\sin \theta_{\mathbf{v}}}, \\
\left|\mathbf{r}-\mathbf{r}_{>}\right|=\frac{\sqrt{\rho^{2}+\rho_{0}^{2}+2 \rho \rho_{0} \sin \phi_{\mathbf{r}}}}{\sin \theta_{\mathbf{v}}},
\end{gathered}
$$

such that the boundary chemical potential $\Delta \mu$ can be expressed, according to Eq. (49) in the effective mean-free-path model, as

$$
\Delta \mu_{>(<)}^{\sigma}\left(\rho, \phi_{\mathbf{r}}\right)=\mathbf{e}_{\mathbf{v}} \cdot e \mathbf{E} \lambda^{\sigma} D_{e q u,>(<)}^{\sigma} \exp \left[\frac{\mp\left(\sqrt{\rho^{2}+\rho_{0}^{2}+2 \rho \rho_{0} \sin \phi_{\mathbf{r}}} / \sin \theta_{\mathbf{v}}-\rho_{<(>)}\right)}{\lambda^{\sigma}}\right],
$$

where $\rho_{>}=2 \rho_{0} \cos \left(\pi / 2-\phi_{>}\right) / \sin \theta_{\mathbf{v}}$ and $\rho_{<}=0$. Substituting Eq. (60) into Eq. (57) easily verifies that Eq. (60) in the effective mean-free-path model is the general solution of Eq. (57) in the Boltzmann transport equation model. Given boundary conditions, the equilibrium diffusion parameter $D_{\text {equ },>(<)}^{\sigma}$ can be undetermined.

\section{CONCLUSIONS}

The solutions for the electron distributions $f_{\mathbf{v}}^{\sigma}(\varepsilon, \mathbf{r})$ in multilayered systems with realistic boundary conditions and with various shapes can be derived from the effective meanfree-path model, which are equivalent to those obtained from the Boltzmann transport equation model. The electron distribution $f_{\mathbf{v}}^{\sigma}(\varepsilon, \mathbf{r})$ is related to the effective mean free path $\lambda_{e f f, \mathbf{v}}^{\sigma}(\mathbf{r})$ and to the effective diffusive scattering specified by $D_{e f f}$. The exponentially decaying $D_{e f f}$ is the maximum of the equilibrium diffusion parameter $D_{e q u, \mathbf{v}}^{\sigma}$, and decays exponentially as the ratio of the distance between the diffusive source and the transport electron to the mean free path $\lambda^{\sigma}$ increases. The equilibrium diffusion parameter $D_{e q u, \mathbf{v}}^{\sigma}$ depends on the given boundary conditions. It should be noted the boundary conditions should be modified when there exists the asymmetry in the different mean free paths between two adjacent materials. This mismatch induces a directional diffusive scattering, which can be specified by the directional modified diffusion parameters in Eqs. (37) and (38).

Unlike the differential equation in the Boltzmann transport equation model, the physical equation in the effective mean-free-path model is an algebraic equation, which is related to the equilibrium diffusion parameter $D_{e q u, \mathbf{v}}^{\sigma}$. Unlike the undetermined mathematical coefficients in the Boltzmann transport equation model, the equilibrium diffusion parameter $D_{e q u, \mathbf{v}}^{\sigma}$ has its physical meaning. Moreover, by use of injecting an incident inverse-transport electron into the system, the transport diagrams like those in Figs. 5 and 6 can be drawn, and then $D_{e q u, \mathbf{v}}^{\sigma}$ can be determined in a diagrammatical way. Therefore, the determination of $D_{e q u, \mathbf{v}}^{\sigma}$ is the diagrammatic derivation with physical insight, rather than the tedious mathematical derivation in the Boltzmann transport equation model. Besides, since $D_{e q u, \mathbf{v}}^{\sigma}$ is associated with the superposition of the determinations to specify all diffusive scatterings, the obtained $D_{e q u, \mathbf{v}}^{\sigma}$ is analytic. The value of $D_{e q u, \mathbf{v}}^{\sigma}$ is expressed as a combination of the corresponding individual physical variables, including the mean free paths, the thickness, the incident angle, the interfacial diffusion parameters, the nondiffusive reflection parameters, and the nondiffusive transmission parameters. The analytic $D_{e q u, \mathbf{v}}^{\sigma}$ is useful in determining the factor that dominates the electron transport and in understanding the reason that the electron transport is sensitive to all interfaces and two outer surfaces, for example the electron transport in a magnetic multilayer under a different applied magnetic field. Furthermore, $D_{e q u, \mathbf{v}}^{\sigma}$ is convergent due to the exponentially decaying traveling factors $t_{\mathbf{v}}^{\sigma}$ in Eq. (17). The convergent $D_{e q u, \mathbf{v}}^{\sigma}$ is useful in writing programs to perform numerical simulations.

Equations (12), (13), (15), (16), (17), (22), (37), and (38) as well as the diagrammatical method are the essence of the effective mean-free-path model. The advantage of the effective mean-free-path model lies in the fact that the solutions for the electron distributions can be directly and easily written down given only the boundary conditions. The obtained solutions have four favorable features: they are analytic, con- 
vergent, diagrammatical, and they have clear physical interpretations. Not only from mathematical structures but also from physical understandings, we can say that the effective mean-free-path model is much more useful than the conventional Boltzmann transport equation model.

\section{ACKNOWLEDGMENT}

This research was supported by the National Science Council of Taiwan under Grant No. NSC93-2112-M-002011.
${ }^{1}$ P. Grünberg, R. Schreiber, Y. Pang, M. B. Brodsky, and H. Sowers, Phys. Rev. Lett. 57, 2442 (1986); G. Binasch, P. Grünberg, F. Saurenbach, and W. Zinn, Phys. Rev. B 39, 4828 (1989); M. N. Baibich, J. M. Broto, A. Fert, F. Nguyen Van Dau, F. Petroff, P. Etienne, G. Creuzet, A. Friederich, and J. Chazelas, Phys. Rev. Lett. 61, 2472 (1988).

${ }^{2}$ S. S. P. Parkin, N. More, and K. P. Roche, Phys. Rev. Lett. 64, 2304 (1990).

${ }^{3}$ Spin Electronics, edited by Michael Ziese and Martin J. Thornton (Springer, New York, 2000).

${ }^{4}$ R. E. Camley and J. Barnas, Phys. Rev. Lett. 63, 664 (1989).

${ }^{5}$ J. Barnas, A. Fuss, R. E. Camley, P. Grünberg, and W. Zinn, Phys. Rev. B 42, 8110 (1990).

${ }^{6}$ P. M. Levy, S. Zhang, and A. Fert, Phys. Rev. Lett. 65, 1643 (1990); S. Zhang and P. M. Levy, J. Appl. Phys. 69, 4786 (1991); P. M. Levy, Solid State Phys. 47, 367 (1994); P. M. Levy and S. Zhang, J. Magn. Magn. Mater. 151, 315 (1995).

${ }^{7}$ X. G. Zhang and W. H. Butler, Phys. Rev. B 51, 10085 (1995); P. Zahn, I. Mertig, M. Richter, and H. Eschrig, Phys. Rev. Lett. 75, 2996 (1995).
${ }^{8}$ M. A. M. Gijs and G. E. W. Bauer, Adv. Phys. 46, 285 (1997).

${ }^{9}$ G. D. Mahan, Many-Particle Physics (Plenum, New York, 1981).

${ }^{10}$ K. Fuchs, Proc. Cambridge Philos. Soc. 34, 100 (1938).

${ }^{11}$ E. H. Sondheimer, Philos. Mag., Suppl. 1, 1 (1952).

${ }^{12}$ S. Zhang and P. M. Levy, Phys. Rev. B 57, 5336 (1998).

${ }^{13}$ A. Shapiro and P. M. Levy, Phys. Rev. B 63, 014419-1 (2000).

${ }^{14}$ T. Valet and A. Fert, Phys. Rev. B 48, 7099 (1993).

${ }^{15}$ H. J. M. Swagten, G. J. Srrijkers, R. H. J. N. Bitter, and W. J. M. de Jonge, IEEE Trans. Magn. 34, 948 (1998).

${ }^{16}$ R. Q. Hood and L. M. Falicov, Phys. Rev. B 46, 8287 (1992).

${ }^{17}$ M. Liu and D.-Y. Xing, Phys. Rev. B 47, 12272 (1993).

${ }^{18}$ Th. G. S. M. Rijks, R. Coehoorn, M. J. M. de Jong, and W. J. M. de Jonge, Phys. Rev. B 51, 283 (1995).

${ }^{19}$ S.-P. Chen and C.-R. Chang, J. Magn. Magn. Mater. 272-276, 1180 (2004).

${ }^{20}$ M. Rubinstein, Phys. Rev. B 50, 3830 (1994).

${ }^{21}$ C.-R. Chang and K.-H. Lo, J. Appl. Phys. 80, 6888 (1996).

${ }^{22}$ P. Rennert and A. Brzezinski, Phys. Rev. B 52, 1612 (1995).

${ }^{23}$ H. E. Comblong, P. M. Levy, and S. Zhang, Phys. Rev. B 51, 16052 (1995). 